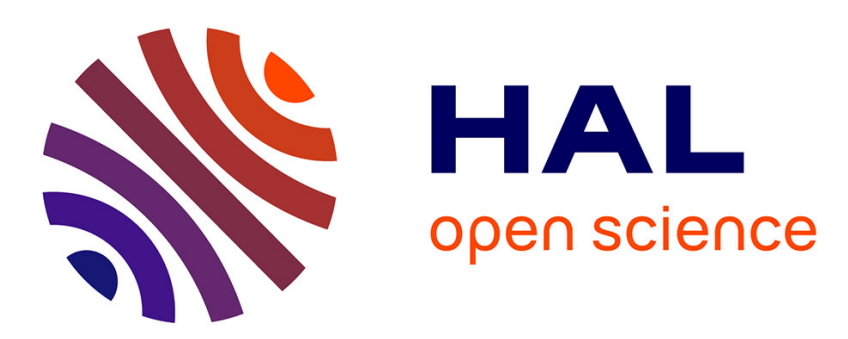

\title{
A Hierarchical Model Predictive Control Framework for On-road Formation Control of Autonomous Vehicles
}

\author{
Xiangjun Qian, Arnaud de La Fortelle, Fabien Moutarde
}

\section{To cite this version:}

Xiangjun Qian, Arnaud de La Fortelle, Fabien Moutarde. A Hierarchical Model Predictive Control Framework for On-road Formation Control of Autonomous Vehicles. 2016 IEEE Intelligent Vehicle Symposium, Jun 2016, Göteborg, Sweden. hal-01298637v2

\section{HAL Id: hal-01298637 \\ https://hal.science/hal-01298637v2}

Submitted on 20 Jul 2016

HAL is a multi-disciplinary open access archive for the deposit and dissemination of scientific research documents, whether they are published or not. The documents may come from teaching and research institutions in France or abroad, or from public or private research centers.
L'archive ouverte pluridisciplinaire $\mathbf{H A L}$, est destinée au dépôt et à la diffusion de documents scientifiques de niveau recherche, publiés ou non, émanant des établissements d'enseignement et de recherche français ou étrangers, des laboratoires publics ou privés. 


\section{A Hierarchical Model Predictive Control Framework for On-road Formation Control of Autonomous Vehicles}

\author{
Xiangjun Qian
}

\author{
Arnaud de La Fortelle
}

\author{
Fabien Moutarde
}

relative orientations/distances from the leader. The virtual structure approach [7], [8] considers the formation as a rigid structure. Robots are regarded as nodes in the structure in which the trajectory of the structure is first calculated and then transformed to the reference trajectories of individual robots. In behavioral approach [9], robots are prescribed with several behaviors, notably goal seeking, local formation keeping, collision avoidance, etc.. The control of each individual robot is a weighted average of the control for each behavior. The global group behavior emerges from the behaviors of individual vehicles.

The literature in robot formation control lays a solid foundation for the convoy control problem of multiple autonomous vehicles. However, unique challenges exist for our problem. Firstly, vehicles are constrained to move in a highly structured environment (multi-lane road with lane markings). Thus the formation must adapt to the road shape and vehicles must avoid departing from their lanes. Secondly, each individual vehicle as well as the entire convoy must respect traffic rules and interact with other traffic participants accordingly. Thirdly, each vehicle must have a complete controller for autonomous driving, ruling out completely centralized approaches. This requirement ensures that a vehicle can still autonomously drive even if it loses communication with the convoy.

There exists some previous work that tackles the convoy control problem of autonomous vehicles on the road. Kato et al. [10] consider a specific convoy problem with 5 vehicles spreading over two lanes using a leader-following approach. In [4], a distributed graph-based convoy control algorithm is proposed. Each vehicle memorizes and tracks its neighborhood. The local control input is calculated using the Laplacian graph method. The advantage of this method is that it is fully decentralized. However, the formation is limited to a rectangle shape and the vehicle controller is in its simplest form.

This paper proposes and validates a novel on-road convoy control algorithm. We adopt a hierarchical approach to separate the convoy control problem into a high-level virtual structure control problem and a low-level vehicle control problem. At high-level, we consider the convoy formation as a rigid virtual structure. We assume that the virtual structure is uni-dimensionally evolving along a reference path, taking into account speed limits, road curvatures and other traffic participants. A MPC controller is adopted to generate the reference trajectory for the virtual structure. This trajectory is then transformed into the reference trajectories of individual vehicles considering longitudinal and lateral offsets of vehi-

\footnotetext{
Xiangjun Qian, Arnaud de La Fortelle and Fabien Moutarde are with MINES ParisTech, PSL - Research University, Centre for Robotics, 60 Bd St Michel, 75006 Paris, France \{xiangjun.qian, arnaud.de_la_fortelle, fabien.moutarde\}@mines-paristech. fr

This research was supported by the European FP7 project AutoNet2030 (Grant Agreement NO. 610542).
} 


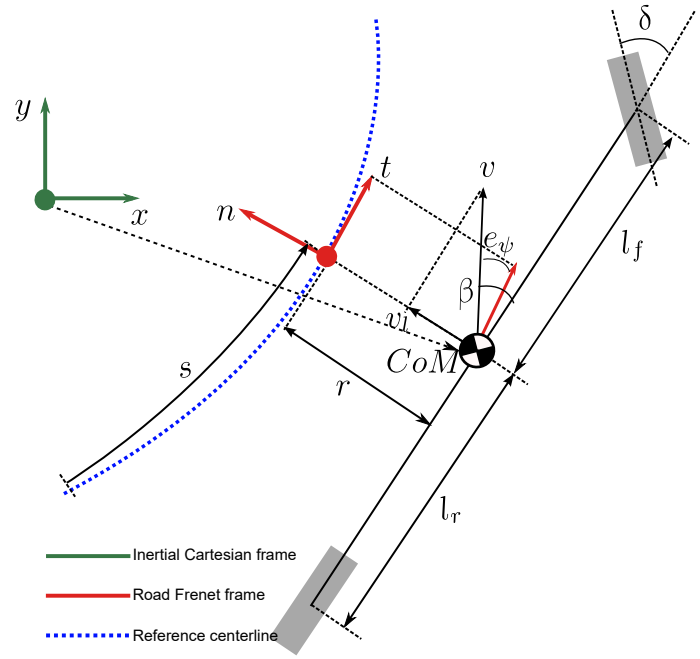

Fig. 1: Kinematic bicycle model in Frenet frame.

cles. At low-level, we propose a complete non-linear MPC controller for autonomous driving of individual vehicles. Behaviors such as lane-keeping and collision avoidance can be readily integrated into the MPC. The cost function of the MPC is designed to penalize the deviation from a formation keeping reference trajectory.

The paper is structured as follows. In Section II, we present the modeling of the convoy control problem. Section III and Section IV present the formulations of the trajectory planning and the trajectory tracking problems, respectively. Section V validates our approach through simulations and Section VI closes the paper with conclusion.

\section{Modeling}

Next we introduce the modeling of the convoy control problem. In particular, Section II-A uses a kinematic bicycle model in the Frenet frame to describe the vehicle dynamics. Section II-B presents the modeling of multi-lane road as a set of parallel curves. Section II-C describes the virtualstructure-based convoy design. Section II-D classifies onroad objects and describes the handling strategies.

\section{A. Vehicle Model}

The car model is developed based on the kinematic bicycle model [11], which strikes a good balance between completeness and complexity. As we focus on on-road autonomous driving, in which vehicle motion is highly constrained by the road geometry, we re-express the kinematic bicycle model in the Frenet frame with respect to the centerline of the lane (Fig.1). This Frenet frame approach is also discussed and applied to different vehicle dynamics models in [12], [13].
The model is given as:

$$
\begin{aligned}
& \dot{s}=v \cos \left(e_{\psi}\right)\left(\frac{1}{1-r \kappa(s)}\right), \\
& \dot{r}=v \sin \left(e_{\psi}\right), \\
& \dot{e}_{\psi}=\dot{\beta}+\frac{v}{l_{r}} \sin (\beta)-v \cos \left(e_{\psi}\right)\left(\frac{\kappa(s)}{1-r \kappa(s)}\right), \\
& \dot{v}=a, \\
& \dot{\delta}=\omega,
\end{aligned}
$$

where $\beta=\tan ^{-1}\left(\frac{l_{r}}{l_{f}+l_{r}} \tan (\delta)\right) . s$ and $r$ are the coordinates of the Center of Mass (CoM) in the road-following Frenet frame. $s$ represents the curvilinear coordinate of the vehicle along the centerline and $r$ is the lateral offset. $e_{\psi}$ is the heading alignment error of the with regard to the CoM centerline and $v$ is the vehicle speed. $l_{r}$ and $l_{f}$ represent the distances from the CoM to the rear and the front axles. $\beta$ is the difference between the angle of the CoM and the heading. $\kappa(s)$ is the curvature of the centerline expressed as a function of $s . \delta$ is the steering angle. $a$ and $\omega$ are two control inputs: acceleration and angular velocity.

\section{B. Road Model}

We give a formal description of multi-lane road. A road with $\mathcal{L}$ lanes is modelled as a collection of parallel curves $\Gamma_{j \in\{1, \ldots, \mathcal{L}\}}$. We express the curve $\Gamma_{j}$ in parametric form as a function of its curvilinear coordinate $s_{j}$ in a 2D Cartesian plane: $\Gamma_{j}\left(s_{j}\right) \in \mathbb{R}^{2}$. Two curves $\Gamma_{j+1}$ and $\Gamma_{j}$ are said to be parallel as they satisfy

$$
\Gamma_{j+1}\left(s_{j}\right)=\Gamma_{i}\left(s_{j}\right)+h \overrightarrow{\boldsymbol{n}}\left(s_{j}\right),
$$

where $h$ is the signed distance of two curves and $\vec{n}$ is a unitary vector orthogonal to $\dot{\Gamma}_{j}\left(s_{j}\right)$ such that the determinant of two vectors are positive: $\operatorname{det}\left(\dot{\Gamma}_{j}\left(s_{j}\right), \overrightarrow{\boldsymbol{n}}\left(s_{j}\right)\right)>0$.

In what follows, we mention two properties of our road model, which will be useful in the rest of the paper.

1) Curvature: The curvature of the curve $\Gamma_{j}$ at coordinate $s_{j}$ reads $\kappa_{j}\left(s_{j}\right)=\dot{x}\left(s_{j}\right) \ddot{y}\left(s_{j}\right)-\dot{y}\left(s_{j}\right) \ddot{x}\left(s_{j}\right)$. The curvature of the parallel curve $\Gamma_{j+1}$ parametrized by coordinate $s_{j}$ of curve $\Gamma_{j}$ reads

$$
\kappa_{j+1}\left(s_{j}\right)=\frac{\kappa_{j}\left(s_{j}\right)}{1-h \kappa_{j}\left(s_{j}\right)} .
$$

Note that a singularity exists for Eq. (3) when 1 $h \kappa_{j}\left(s_{j}\right)=0$. We assume that $h \kappa_{j}\left(s_{j}\right)<1$, conforming to the normal road geometry.

2) Change of reference frame: In a multi-lane road setting, the curvilinear coordinate $s_{j}$ of a vehicle with regard to curve $\Gamma_{j}$ differs from its curvilinear coordinate $s_{j+1}$ with regard to curve $\Gamma_{j+1}$. The two coordinates satisfies the following relation:

$$
s_{j+1}=\int_{0}^{s_{j}}\left|1-h \kappa_{j}\left(\varepsilon_{j}\right)\right| d \varepsilon_{j}
$$




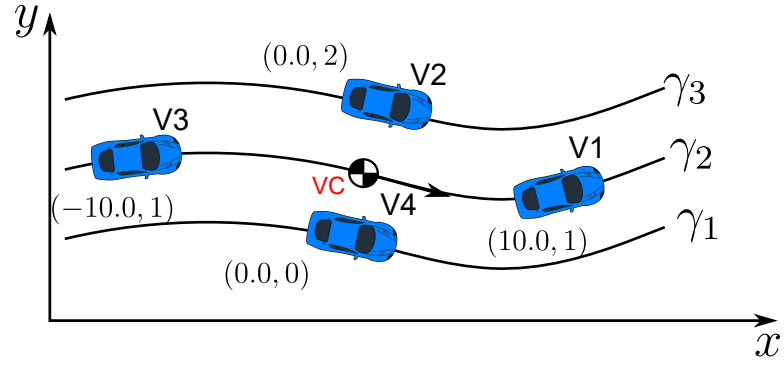

Fig. 2: Convoy formation defined by curvilinear longitudinal offset and lane number

\section{Convoy Model}

In the virtual-structure-based approach, the convoy is regarded as a rigid body with place-holders fixed in the formation to represent the desired positions [7]. Let $\mathcal{V}$ denote the number of member vehicles in the formation. We use $i \in\{1, \ldots, \mathcal{V}\}$ to label member vehicles and $r_{i}$ to label the desired lane of vehicle $i$, for example, $r_{i}=j$ if vehicle $i$ is assigned to lane $j$. To determine the shape of the virtual structure, we introduce a reference point called the virtual center that evolves uni-dimensionally on a centerline $\Gamma_{c}$. It is appropriate to consider the dynamics of the virtual center using a simple double integrator model:

$$
\begin{aligned}
& \dot{s_{c}}=v_{c}, \\
& \dot{v}_{c}=a_{c},
\end{aligned}
$$

where $s_{c}$ and $v_{c}$ are curvilinear coordinate and speed on the curve $\Gamma_{c} . a_{c}$ is the acceleration.

Knowing the current position of the virtual center, the desired position of a vehicle $i$ can then be defined by offseting the position of the virtual center, formally described by the tuple $\left(\Delta s_{i, c}, r_{i}\right) \in \mathbb{R} \times \mathbb{N}^{+}$, where $\Delta s_{i, c}$ is the desired longitudinal offset of the vehicle $i$ with regard to the virtual center in the $\Gamma_{c}$ frame, $r_{i}$ is the lane label of the vehicle's desired lane. Fig. 2 provides an illustrative example defining a diamond-shaped convoy. The virtual center is assigned to lane $\Gamma_{2}$. Vehicles V1 and V3 are assigned to lane $\Gamma_{2}$ with respectively $10 \mathrm{~m}$ and $-10 \mathrm{~m}$ offsets from the virtual center. Vehicles V2 and V4 are assigned respectively to $\Gamma_{3}$ and $\Gamma_{1}$ with 0 longitudinal offset.

However, in local vehicle control, we consider the motion with regard to the vehicle's own reference frame $\Gamma_{r_{i}}$. The longitudinal coordinate in the $\Gamma_{c}$ frame then needs to be transformed to the coordinate in the $\Gamma_{r_{i}}$ frame using the Eq. (4).

With the above formulation, a virtual-structure-based vehicle convoy can be uniquely defined by giving the position of the virtual center and the tuple $\left(\Delta s_{i, c}, r_{i}\right)$ for each member vehicle.

\section{Object Model}

Vehicle convoys must respect traffic rules and interact with other traffic participants and obstacles accordingly. This requirement demands a proper modeling of on-road objects. We propose to classify on-road objects into two categories:

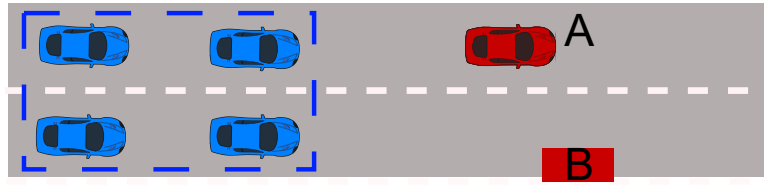

Fig. 3: Illustration of on-road objects.

- Lane-Blocking Obstacles (LBOs). Static or dynamic obstacles that block one or more lanes. An example is given in Fig. 3 where the left lane is blocked by vehicle A.

- Non-Blocking Obstacles (NBOs). Static or dynamic obstacles that only block a small part of a lane. They can be dealt with by swerve maneuvers of member vehicles. The object B in Fig. 3 illustrates an avoidable obstacle on the border of the road.

LBOs and NBOs need to be handled differently. A vehicle convoy cannot overtake LBOs unless it changes its formation. Thus LBOs must be handled in convoy trajectory planning level. An adequate convoy speed should be planned to keep safe distances from LBOs. On the other hand, NBOs should be ignored at high level and left for individual vehicles to handle, as local swerve maneuvers are sufficient.

Remark that LBOs may become NBOs and vice versa depending on the trajectory of obstacles and the changes of convoy formation. These problems are not considered in this paper.

\section{MPC BASED TRAJECTORY GENERATION FOR THE VIRTUAL CENTER}

Next we present how the trajectory of the virtual center can be generated using MPC. Let $\boldsymbol{\xi}_{c}=\left[s_{c}, v_{c}\right] \in \boldsymbol{\Xi}_{c}$ be the state of the virtual center and $\boldsymbol{u}_{c}=\left[a_{c}\right] \in \boldsymbol{U}_{c}$ be the input. We assume that at a sampling time $t=t_{0}$ a desired trajectory for the interval $t \in\left[t_{0}, t_{0}+T_{c}\right]$ should be provided. Consider the following cost function:

$$
\begin{aligned}
& \mathcal{J}_{c}\left(\boldsymbol{\xi}_{c}, \boldsymbol{u}_{c}\right)= \\
& \quad \int_{t_{0}}^{t_{0}+T_{c}}\left(w_{1}\left(v_{c}(t)-v_{c, \text { desire }}\right)^{2}+w_{2} a_{c}^{2}(t)\right) d t,
\end{aligned}
$$

where the first term penalizes the deviation of convoy speed from the desired speed $v_{c, \text { desire }}$ and the second term penalizes the control effort. These two terms are weighted through the tuning parameters $w_{1}$ and $w_{2}$.

At each sampling time $t=t_{0}$, we solve the following constrained optimization problem:

$$
\min _{\boldsymbol{u}_{c}} \mathcal{J}_{c}\left(\boldsymbol{\xi}_{c}, \boldsymbol{u}_{c}\right),
$$

subj. to $\forall t \in\left[t_{0}, t_{0}+T_{c}\right]$,

$$
\begin{aligned}
& \dot{\boldsymbol{\xi}}_{c}(t)=f_{c}\left(\boldsymbol{\xi}_{c}(t), \boldsymbol{u}_{c}(t)\right), \\
& \boldsymbol{\xi}_{c}(t) \in \boldsymbol{\Xi}_{c}, \boldsymbol{u}_{c}(t) \in \boldsymbol{U}_{c}, \\
& v_{c}^{2}(t) \kappa_{c}\left(s_{c}(t)\right) \leq \bar{a}_{c, l a t}, \\
& f_{c}\left(\boldsymbol{\xi}_{c}(t), \boldsymbol{\xi}_{o, c}(t)\right) \leq 0, \forall o,
\end{aligned}
$$


Eq. (7a) is the compactly written form of the virtual center model (5). (7b) enforces bound constraints on state and input, such as speed limit and acceleration bounds. The lateral acceleration of convoy is limited through $(7 \mathrm{c})$. Constraint (7d) is used to avoid collision with LBOs where $\boldsymbol{\xi}_{o, c}$ is the state of a LBO $o$ in $\Gamma_{c}$ frame. A possible design of this constraint can be:

$$
s_{c}(t)+v_{c}(t) T_{\text {desire }}+\epsilon \leq s_{o, c}(t)
$$

where $s_{o, c}(t)$ is the estimated longitudinal trajectory of LBO $o$ in $\Gamma_{c}$ frame, $T_{\text {desire }}$ is the desired constant time gap and $\epsilon$ is the minimal standstill distance. This constraint forces the virtual center to keep a constant time gap from the LBO.

Solving the optimization problem results in an optimal trajectory $\boldsymbol{\xi}_{c}^{*}(t)$ for the horizon $\left[t_{0}, t_{0}+T_{c}\right]$. This trajectory can then be transformed to reference trajectories to be tracked by member vehicles. Problem (7) is solved repeatedly at a sampling rate of $1 / \Delta T_{c}$, incorporating new measurements of the environment.

\section{MPC BASED TRAJECTORY TRACKING CONTROL FOR VEHICLES}

In this section, we consider the tracking control design for an arbitrary member vehicle $i$ of the convoy. As the tracking control problem is always considered in the reference frame $\Gamma_{r_{i}}$, for the sake of simplicity, we drop out the subscript $r_{i}$ so that $s_{i, r_{i}}$ becomes $s_{i}$, and apply similar rules to other state variables. We denote the vehicle state $\boldsymbol{\xi}_{i}=$ $\left[s_{i}, e_{l, i}, e_{\psi, i}, v_{i}, \delta_{i}\right] \in \boldsymbol{\Xi}_{i}$ and the input $\boldsymbol{u}_{i}=\left[a_{i}, \omega_{i}\right] \in \boldsymbol{U}_{i}$.

Consider the following cost function in least-square form:

$$
\begin{aligned}
\mathcal{J}_{i}\left(\boldsymbol{\xi}_{i}, \boldsymbol{u}_{i}\right)= & \int_{0}^{T_{i}}\left(\left\|\boldsymbol{\xi}_{i}(t)-\boldsymbol{\xi}_{i, r e f}(t)\right\|_{Q}^{2}\right. \\
& \left.+\left\|\boldsymbol{u}_{i}(t)-\boldsymbol{u}_{i, r e f}(t)\right\|_{R}^{2}\right),
\end{aligned}
$$

where $T_{i}$ is the prediction horizon. $\boldsymbol{\xi}_{i, r e f}(t)=$ $\left[s_{i, r e f}(t), 0,0,0,0\right]$ is the formation keeping reference trajectory from the high level MPC and $\boldsymbol{u}_{i, r e f}(t)$ is the reference input usually set to $\mathbf{0}$. $Q=\operatorname{diag}\left(q_{1}, \ldots q_{5}\right)$ and $R=\operatorname{diag}\left(r_{1}, r_{2}\right)$ are two positive diagonal weighting matrices. It is shown in [14] that the stability of MPC can be guaranteed by properly choosing a final state constraint and a final cost. However, in our problem it is difficult to fix a final state or a final state set due to the existence of obstacles. An empirical trial-and-error approach is used to find a long-enough horizon $T_{i}$ that is stabilizing.

At each sampling time $t=t_{0}$, we solve the following constrained optimization problem:

$$
\min _{\boldsymbol{u}_{i}} \mathcal{J}_{i}\left(\boldsymbol{\xi}_{i}, \boldsymbol{u}_{i}\right)
$$

subj. to $\forall t \in\left[t_{0}, t_{0}+T_{c}\right]$,

$$
\begin{aligned}
& \dot{\boldsymbol{\xi}}_{i}(t)=f_{i}\left(\boldsymbol{\xi}_{i}(t), \boldsymbol{u}_{i}(t)\right), \\
& \boldsymbol{\xi}_{i}(t) \in \boldsymbol{\Xi}_{i}, \boldsymbol{u}_{i}(t) \in \boldsymbol{U}_{i}, \\
& v_{i}(t)\left(\dot{\beta}_{i}(t)+\frac{v_{i}(t)}{l_{r}} \sin \left(\beta_{i}(t)\right)\right) \in\left[-\bar{a}_{i, l a t}, \bar{a}_{i, l a t}\right], \\
& f_{i}\left(\boldsymbol{\xi}_{i}(t), \boldsymbol{\xi}_{o}(t)\right) \leq 0, \forall o .
\end{aligned}
$$

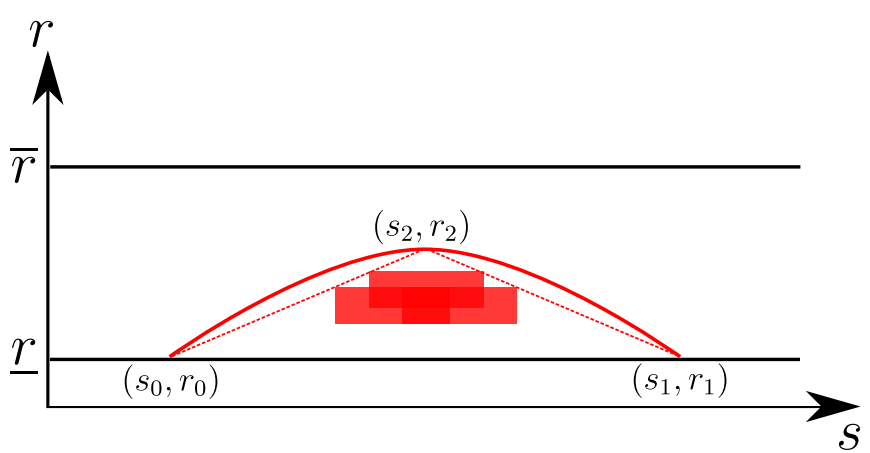

Fig. 4: Approximation of obstacle region by a parabola.

Eq. (10a) is the compactly written form of vehicle model (1). (10b) enforces bound constraints on state and input, such as speed limit, maximal lateral offset, maximal steering angle, acceleration bounds, etc. (10c) bounds the lateral acceleration of the vehicle. Constraint (10d) ensures collision avoidance with NBOs where $\boldsymbol{\xi}_{o}=\left[s_{o}, r_{o}\right]$ is the state of a NBO $o$ in the $\Gamma_{r_{i}}$ frame. Here we provide some details on the constraint design for NBOs. Consider an obstacle described as a polygon in Fig 4. If directly integrated into the optimization problem, the resulting constraint is not differentiable and it creates two homotopy classes of feasible trajectories. We choose to assign the obstacle to its nearest boundary (in this case the lower one), augment the obstacle region to a triangle and use a parabolic constraint in the form of $r \geq a s^{2}+b s+c$ to approximate the obstacle region. The unknown coefficients $a, b, c$ can be calculated by solving the following linear system.

$$
\left(\begin{array}{lll}
s_{0}^{2} & s_{0} & 1 \\
s_{1}^{2} & s_{1} & 1 \\
s_{2}^{2} & s_{2} & 1
\end{array}\right)\left(\begin{array}{l}
a \\
b \\
c
\end{array}\right)=\left(\begin{array}{l}
r_{0} \\
r_{1} \\
r_{2}
\end{array}\right)
$$

The solution of optimization problem (10) is the optimal control input $\boldsymbol{u}_{i}^{*}(t), t \in\left[t_{0}, t_{0}+T_{i}\right]$ as well as the associated optimal trajectory $\boldsymbol{\xi}_{i}^{*}$. Let $\Delta T_{i}$ be the replanning interval. Then only the fraction $\left[t_{0}, t_{0}+\Delta T_{i}\right]$ of $\boldsymbol{u}_{i}^{*}(t)$ will be fed into the vehicle low-level controller and the problem is solved again at $t=t_{0}+\Delta T_{i}$.

\section{EXPERIMENTS AND RESULTS}

Experiments are carried out using the high-fidelity robotic simulator Webots [16]. Webots provides a realistic vehicle model with steering dynamic response and friction of tires. The proposed convoy algorithm is implemented in Webots using $\mathrm{C}++$ language and optimization problems are solved using ACADO toolkit [17]. The integrated simulation runs on an Ubuntu machine with Intel Core i7 CPU clocked at $3.40 \mathrm{GHz}$ and $32 \mathrm{~GB}$ RAM.

In our experiments, we consider a diamond formation as in Fig. 2. The trajectory generation module is implemented in the foremost vehicle (V1). Note that it can also be implemented in other vehicles or even in a remote server. Vehicles are mounted with localization system and communication devices. Vehicle localization has a $0.2 \mathrm{~m}$ precision and communication devices are assumed to be 
delay-free for this experiment (delays will be considered in the future research). The trajectory of the virtual center is disseminated to all vehicles through communication. The trajectory tracking module is implemented in each vehicle. Here we only consider homogeneous convoys, in which all vehicles have the same characteristics. Convoy parameters are given as: $0 \leq v_{c} \leq 15 \mathrm{~m} / \mathrm{s}, v_{c, \text { desire }}=12 \mathrm{~m} / \mathrm{s}$, $\left|a_{c}\right| \leq 1.5 \mathrm{~m} / \mathrm{s}^{2}$ and $\bar{a}_{c, l a t}=1 \mathrm{~m} / \mathrm{s}^{2}$. The parameters used for trajectory generation of the virtual center is $w_{1}=1$, $w_{2}=4, T_{c}=10 \mathrm{~s}$ and $\Delta T_{c}=0.256 \mathrm{~s}$. Vehicle parameters are given as: $0 \leq v_{i} \leq 20 \mathrm{~m} / \mathrm{s},\left|a_{i}\right| \leq 2.5 \mathrm{~m} / \mathrm{s}^{2},\left|\bar{a}_{i, l a t}\right|=$ $2.5 \mathrm{~m} / \mathrm{s}^{2},|\delta| \leq 0.64 \mathrm{rad},|\omega| \leq 0.05 \mathrm{rad} / \mathrm{s}, l_{r}=1.30 \mathrm{~m}$ and $l_{f}=1.70 \mathrm{~m}$. The parameters used for trajectory tracking are $Q=\operatorname{diag}(15,8,1000,0,20), R=\operatorname{diag}(1,600), T_{i}=5 \mathrm{~s}$ and $\Delta T_{i}=0.128 \mathrm{~s}$.

We consider three sets of experiments, respectively:

- convoy control while driving in a road with straight and curvy segments,

- convoy control while driving in a road with a speedvarying LBO in front,

- convoy control while driving in a road with multiple NBOs.

In the first experiment, we aim to demonstrate the formation keeping capability of our approach under localization noise. Vehicle convoy starts from zero speed and non-zero errors in formation. Fig. 5a presents the trajectories of convoy vehicles. Fig. $5 \mathrm{~b}$ provides the evolution of formation errors defined as the difference between the actual vehicle position and the desired position. We observe that at the beginning, vehicles quickly converge to the desired formation. The formation errors of all vehicles decrease to the level of localization uncertainty in less than $5 \mathrm{~s}$. Formation errors increase slightly (while still less than $1 \mathrm{~m}$ ) when convoy enters and exits the curvy segment. Fig.5c shows the evolution of speed of the virtual center and individual vehicles. We remark two points: 1) convoy speed is adjusted in the curvy segment to reduce lateral acceleration. 2) Individual vehicle speeds track closely the convoy speed except in the curvy segment, where the vehicle in the inner lane has a smaller speed while the vehicle in the outer lane has a higher speed.

The second experiment aims to verify if the convoy can safely handle speed-varying LBOs. The desired time space is selected as $2 \mathrm{~s}$. Fig. 7 presents the speed curves of the convoy and the LBO in the front and the distance between the virtual center and the LBO. The LBO first decelerates and then accelerates to recover its original speed. We observe that the convoy adapts its speed accordingly. We also observe that a safe distance is always kept.

The third experiment demonstrates the capability of our approach to avoid on-road NBOs. Three NBOs are distributed over two lanes. Vehicle convoy traverses the road segment at a constant speed of $12 \mathrm{~m} / \mathrm{s}$. Trajectories are illustrated in Fig. 8, we observe that the vehicles successfully avoid NBOs while maintaining the formation.

The average computation time for the trajectory generation of the virtual center is $0.033 \mathrm{~s}$, and the standard deviation is

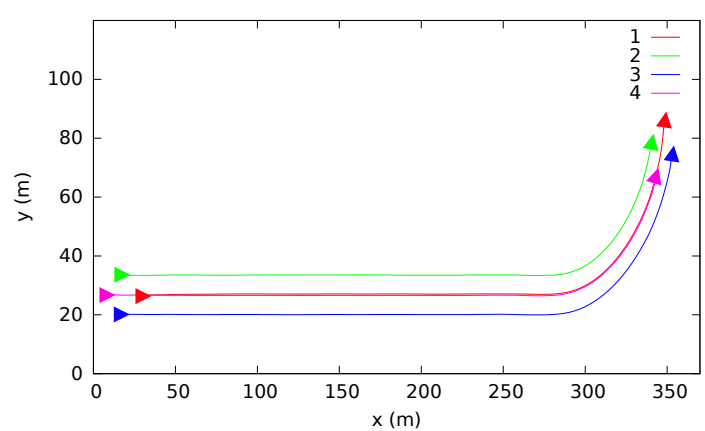

(a)

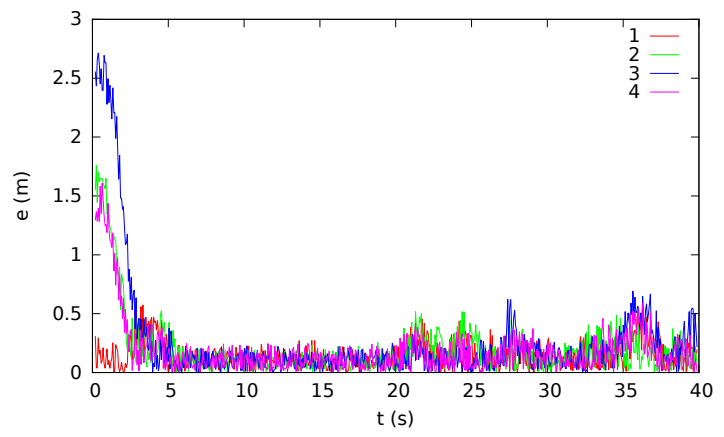

(b)

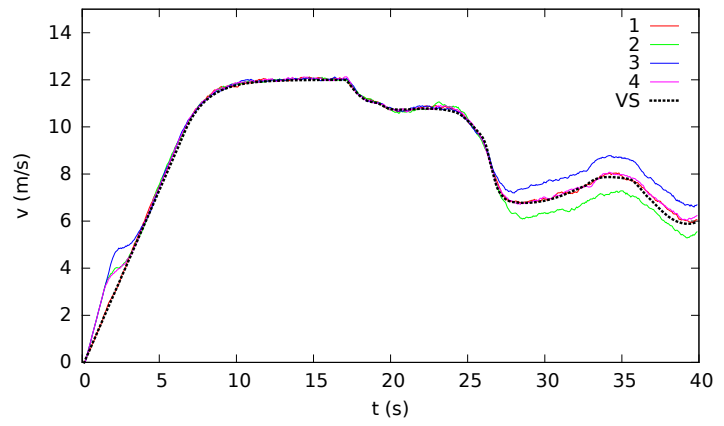

(c)

Fig. 5: First experiment: (a) trajectory of the convoy, (b) formation error and (c) speed.

$0.014 \mathrm{~s}$. For the computation time of trajectory tracking, the average is $0.071 \mathrm{~s}$, and the standard deviation is $0.020 \mathrm{~s}$.

Videos of the three experiments are available on-line ${ }^{1}$ and can also be found in the supplementary material of the submission.

\section{CONCLUSION}

We have presented a hierarchical MPC scheme for on-road convoy control of autonomous vehicles. The high level MPC generates the reference trajectory of the convoy, considering various constraints. The reference trajectory is then communicated to member vehicles with different formation offsets. At low level, a local MPC tracking controller is used to drive each vehicle towards its desired trajectory. Experiments have demonstrated the effectiveness of the approach in handling realistic on-road conditions such as curvy segments, other

\footnotetext{
${ }^{1}$ https://youtu.be/70wMhrYuCTo
} 


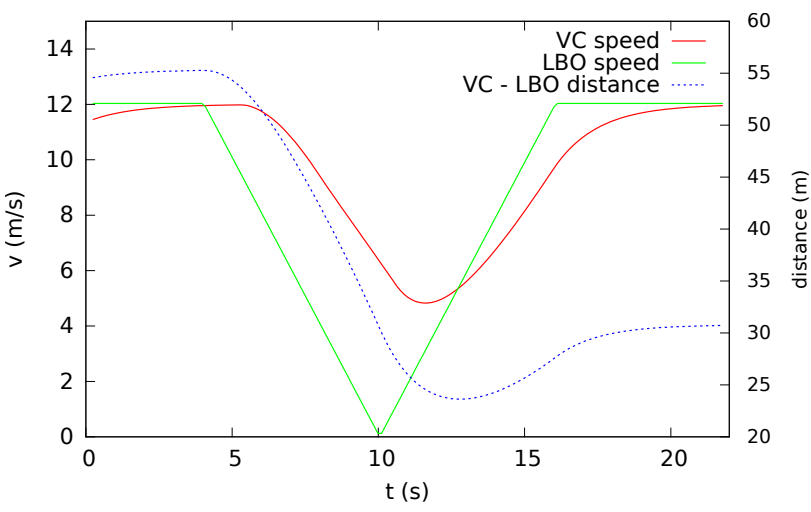

Fig. 6

Fig. 7: Second experiment: speed of the virtual center and LBO, distance from the virtual center to LBO.

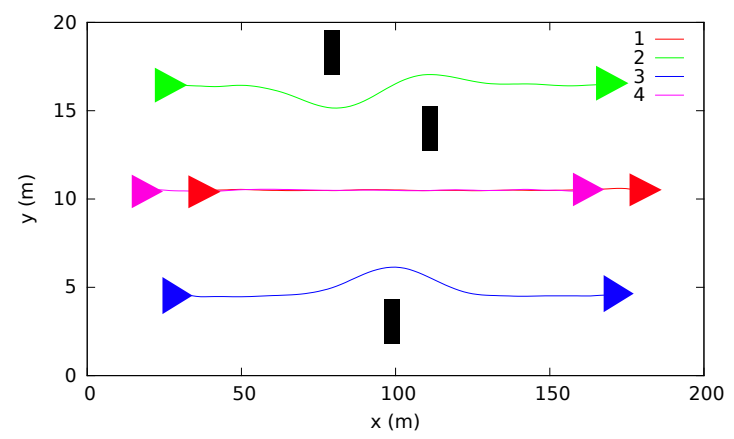

Fig. 8: Third experiment: trajectory of the convoy. Black boxes mark obstacles.

traffic participants and obstacles. The current paper focuses on the modeling of convoy and the surrounding enviroment and the presentation of the components of MPC based scheme. In the future, we will consider more advanced issues such as the incorporation of uncertainties from communication and perception and the dynamic formation modification in merging scenario. Moreover, we plan to implement and demonstrate our scheme in real vehicles under the framework of the European project AutoNET2030 [18].

\section{ACKNOWLEDGMENT}

We thank Florent Altché and Philip Polack for their insightful comments on the paper.

\section{REFERENCES}

[1] S. E. Shladover, C. A. Desoer, J. K. Hedrick, M. Tomizuka, J. Walrand, W.-B. Zhang, D. H. McMahon, H. Peng, S. Sheikholeslam, and N. McKeown, "Automated vehicle control developments in the PATH program," Vehicular Technology, IEEE Transactions on, vol. 40, pp. 114-130, Feb. 1991.

[2] B. J. Harker, "PROMOTE-CHAUFFEUR II \& $5.8 \mathrm{GHz}$ vehicle to vehicle communications system," in Advanced Driver Assistance Systems, 2001. ADAS. International Conference on (IEE Conf. Publ. No. 483), pp. 81-85, 2001.

[3] E. Chan, P. Gilhead, P. Jelinek, P. Krejci, and T. Robinson, "Cooperative control of sartre automated platoon vehicles," in 19th ITS World Congress, Vienna, Austria, pp. 22-26, 2012.
[4] A. Marjovi, M. Vasic, J. Lemaitre, and A. Martinoli, "Distributed graph-based convoy control for networked intelligent vehicles," in Intelligent Vehicles Symposium (IV), 2015 IEEE, pp. 138-143, June 2015.

[5] J. Shao, G. Xie, and L. Wang, "Leader-following formation control of multiple mobile vehicles," Control Theory Applications, IET, vol. 1, pp. 545-552, Mar. 2007.

[6] L. Consolini, F. Morbidi, D. Prattichizzo, and M. Tosques, "Leaderfollower formation control of nonholonomic mobile robots with input constraints," Automatica, vol. 44, no. 5, pp. 1343-1349, 2008.

[7] W. Ren and R. W. Beard, "Virtual structure based spacecraft formation control with formation feedback," AIAA Paper, vol. 4963, 2002.

[8] W. Ren and R. Beard, "Decentralized scheme for spacecraft formation flying via the virtual structure approach," Journal of Guidance, Control, and Dynamics, vol. 27, no. 1, pp. 73-82, 2004.

[9] T. Balch and R. C. Arkin, "Behavior-based formation control for multirobot teams," Robotics and Automation, IEEE Transactions on, vol. 14, no. 6, pp. 926-939, 1998.

[10] S. Kato, S. Tsugawa, K. Tokuda, T. Matsui, and H. Fujii, "Vehicle Control Algorithms for Cooperative Driving with Automated Vehicles and Intervehicle Communications," IEEE Transactions on Intelligent Transportation Systems, vol. 3, no. 3, pp. 155-160, 2002.

[11] J. Kong, M. Pfeiffer, G. Schildbach, and F. Borrelli, "Kinematic and Dynamic Vehicle Models for Autonomous Driving Control Design," pp. $2-7$.

[12] M. Werling, S. Kammel, J. Ziegler, and L. Groll, "Optimal trajectories for time-critical street scenarios using discretized terminal manifolds," The International Journal of Robotics Research, vol. 31, no. September 2015, pp. 346-359, 2012.

[13] T. Weiskircher and B. Ayalew, "Predictive ADAS : A Predictive Trajectory Guidance Scheme for Advanced Driver Assistance in Public Traffic *,"

[14] D. Q. Mayne, J. B. Rawlings, C. V. Rao, and P. O. Scokaert, "Constrained model predictive control: Stability and optimality," $\mathrm{Au}$ tomatica, vol. 36, no. 6, pp. 789-814, 2000.

[15] D. Limon, T. Alamo, and E. Camacho, "Stable constrained mpc without terminal constraint," in American Control Conference, 2003. Proceedings of the 2003, vol. 6, pp. 4893-4898, IEEE, 2003.

[16] O. Michel, "Webots: Professional Mobile Robot Simulation," International Journal of Advanced Robotic Systems, vol. 1, no. BIOROBARTICLE-2004-001, pp. 39-42, 2004.

[17] B. Houska, H. J. Ferreau, and M. Diehl, "ACADO Toolkit - An Open Source Framework for Automatic Control and Dynamic Optimization," Optimal Control Applications and Methods, vol. 32, no. 3, pp. 298312, 2011.

[18] A. de La Fortelle, X. Qian, S. Diemer, F. Moutarde, and S. Bonnabel, "Network of Automated Vehicles: the AutoNet2030 vision," in ITS World Congress, 2014. 